\title{
Favorable control of advanced colon adenocarcinoma with severe bone marrow metastasis: A case report
}

\author{
FUMIYASU HANAMURA ${ }^{1,2}$, YOSHIHIRO SHIBATA ${ }^{1}$, TSUYOSHI SHIRAKAWA ${ }^{2}$, MIYUKI KUWAYAMA ${ }^{2}$, \\ HISANOBU ODA ${ }^{2,3}$, HIROSHI ARIYAMA ${ }^{4}$, KENICHI TAGUCHI $^{5}$, TAITO ESAKI $^{2}$ and EISHI BABA ${ }^{6}$ \\ ${ }^{1}$ Department of Medical Oncology, Fukuoka Wajiro Hospital, Fukuoka 811-0213; ${ }^{2}$ Department \\ of Gastrointestinal and Medical Oncology, National Hospital Organization Kyushu Cancer Center, Fukuoka 811-1395; \\ ${ }^{3}$ Department of Medical Oncology, Saiseikai Fukuoka General Hospital, Fukuoka 810-0001; ${ }^{4}$ Department of Hematology \\ and Oncology, Kyushu University Hospital, Fukuoka 812-8582; ${ }^{5}$ Department of Cancer Biology, National Hospital \\ Organization Kyushu Cancer Center, Fukuoka 811-1395; ${ }^{6}$ Department of Comprehensive Clinical Oncology, \\ Faculty of Medical Sciences, Kyushu University, Fukuoka 812-8582, Japan
}

Received June 1, 2016; Accepted August 25, 2016

DOI: $10.3892 / \operatorname{mco} .2016 .1029$

\begin{abstract}
Colorectal cancer (CRC) has a propensity to metastasize to the liver, lungs and regional abdominal lymph nodes, but rarely to the bone marrow. A 60-year-old man presented to the National Hospital Organization Kyushu Cancer Center with a 4-week history of persistent lower back pain, anorexia and difficulty defecating. Complete blood count revealed severe thrombocytopenia and erythroblastosis, suggesting a hematological malignancy. However, the bone marrow examination demonstrated involvement by a moderately to poorly differentiated adenocarcinoma, but no hematopoietic abnormalities. A computed tomography scan revealed thickening of the wall of the sigmoid colon, with para-aortic, hilar, mediastinal and supraclavicular lymphadenopathy. The patient was thus diagnosed with sigmoid colon adenocarcinoma with lymph node and bone marrow metastasis. Modified FOLFOX6 was promptly initiated, with concurrent therapy for disseminated intravascular coagulation (DIC). An increased number of thrombocytes was observed on day 6 . After 3 cycles of treatment, the patient recovered from DIC and the levels of serum carcinoembryonic antigen and cytokeratin 19 fragment were decreased. Tumor biopsy during colonoscopy following recovery from DIC demonstrated poorly differentiated adenocarcinoma with mucin production, without mutations in the $R A S, B R A F$ or PIK3CA genes, and a cytokeratin (CK) 7-negative, CK20-positive phenotype. The patient has been treated
\end{abstract}

Correspondence to: Professor Eishi Baba, Department of Comprehensive Clinical Oncology, Faculty of Medical Sciences, Kyushu University, 3-1-1 Maidashi, Higashi-ku, Fukuoka 812-8582, Japan

E-mail: e-baba@c-oncology.med.kyushu-u.ac.jp

Key words: colon cancer, bone marrow metastasis, disseminated intravascular coagulation, chemotherapy with chemotherapy for 150 days without disease progression. However, the efficacy of chemotherapy for rarely encountered bone marrow metastasis from CRC is poor. The present case was favorably maintained on chemotherapy and survived for 10 months.

\section{Introduction}

Bone marrow metastasis from malignant solid tumors may be occasionally encountered, particularly with cancers of the breast, stomach and prostate gland (1). Decreased blood cell count and abnormal coagulation status are often observed in cases with bone marrow metastasis from malignant tumors, and disseminated intravascular coagulation (DIC) or thrombotic thrombocytopenic purpura (TTP) occasionally develop. Supportive therapy against the abnormal coagulation status alone is inadequate in patients with DIC precipitated by malignancy, and simultaneous appropriate chemotherapy against the tumor is required to improve the coagulation abnormalities. Specific chemotherapeutic regimens against bone marrow metastasis have not been developed, and the majority of such patients have a poor prognosis (2-4).

Colorectal cancer (CRC) is the third cause of cancer-related mortality worldwide (5). Approximately $40 \%$ of CRC patients present with distant metastases, either at diagnosis or during the course of treatment. Metastasis frequently affects the liver, abdominal lymph nodes, lungs and peritoneum. Systemic chemotherapy is employed as a standard therapy and the effectiveness of chemotherapy has recently improved in association with the use of cytotoxic and molecular-targeted agents.

However, a limited number of CRC cases were reported to clinically exhibit bone marrow metastases (6-10). The majority of these cases displayed hematological disorders, including thrombocytopenia, DIC, TTP and microangiopathic hemolytic anemia. The general condition of the patients tended to be poor and metastasis was also observed in organs other than the bone marrow, such as the liver, lymph nodes and lungs. The efficacy of systemic chemotherapy against CRC with bone 
marrow metastasis appears to be poor, and the prognosis for such patients is unfavourable. Only 10 cases of CRC with bone marrow metastasis have been reported (6) and the common molecular characteristics of these cases have not been identified.

Our patient presented with metastasis of sigmoid colon cancer to the bone marrow and systemic chemotherapy achieved satisfactory disease control. We herein present the immunohistochemical and molecular biological analyses of the primary and metastatic sites in this case and compare the findings with those of previous reports.

\section{Case report}

A 60-year-old man presented with a 4-week history of persistent lower back pain, anorexia and difficulty defecating. The patient visited a local doctor complaining of abdominal pain and blood in the stool. He had a 40-pack-year history of smoking and a drinking history of 1 glass of beer per day; his medical history was otherwise non-significant. The family history included lung and gastric cancer in his father, and pancreatic cancer in his mother. Due to the presence of severe thrombocytopenia and erythroblastosis, suggesting a hematological malignancy, the patient was referred to the National Hospital Organization Kyushu Cancer Center (Fukuoka, Japan) in December, 2014. The Eastern Cooperative Oncology Group performance status (PS) was 2 and the axillary temperature was $37.9^{\circ} \mathrm{C}$. Other vital signs were within the normal range. Lower abdominal pain was present, without subcutaneous hemorrhage. Blood testing revealed a decreased red blood cell count of $3.05 \times 10^{6} / \mu 1$, a decreased hemoglobin level of $9.6 \mathrm{~g} / \mathrm{dl}$, an increased white blood cell of $13.85 \times 10^{3} / \mu 1$ and a decreased platelet count at $5 \times 10^{3} / \mu 1$. Analysis of the coagulation system revealed a prolonged prothrombin time-international normalization ratio (1.39) and increased levels of fibrin/fibrinogen degradation product-P $(209.02 \mu \mathrm{g} / \mathrm{ml})$ and D-dimer $(168.17 \mu \mathrm{g} / \mathrm{ml})$. DIC was diagnosed based on a score of 9 according to the criteria of the Ministry of Health, Labour and Welfare of Japan (11). Serum biochemistry revealed elevated levels of lactate dehydrogenase at 1,359 IU/1 and C-reactive protein at $14.91 \mathrm{mg} / \mathrm{dl}$. The serum levels of carcinoembryonic antigen (CEA; $46.4 \mathrm{ng} / \mathrm{ml})$, soluble interleukin 2 receptor $(1,364 \mathrm{U} / \mathrm{ml})$ and cytokeratin 19 fragment (CYFRA; $78.3 \mathrm{ng} / \mathrm{ml})$ were also elevated.

Although hematological malignancy was suspected, an aspiration biopsy from the bone marrow revealed moderately to poorly differentiated adenocarcinoma, without leukemic cells (Fig. 1A). Immunohistochemical examination revealed that the tumor cells were negative for cytokeratin (CK) 7 and positive for CK20 and caudal type homeobox transcription factor 2 (CDX2). Computed tomography (CT) revealed thickening of the sigmoid colon wall and para-aortic, hilar, mediastinal and right supraclavicular lymphadenopathy (Fig. 2A). Moreover, osteolytic lesions were detected diffusely in all the vertebrae and the ilium. Although colonoscopy was not performed due to severe thrombocytopenia, sigmoid colon cancer with metastases to the lymph nodes and bone marrow was diagnosed. Chemotherapy using a modified FOLFOX6 (mFOLFOX6) regimen for advanced sigmoid colon cancer was promptly initiated, but the dose of the chemotherapeutic agents was reduced to $75 \%$ due to worsening of the thrombocytopenia. Supportive therapies comprising transfusion of thrombocytes and administration of gabexate mesilate to control DIC were performed simultaneously. Bevacizumab was not initially administered due to the risk of hemorrhagic events. The number of thrombocytes increased to $61 \times 10^{3} / \mu 1$ after 3 cycles of chemotherapy and the serum CEA and CYFRA levels decreased to 5.4 and $2.4 \mathrm{ng} / \mathrm{ml}$, respectively. Common Terminology Criteria of Adverse Events v.4.0 grade 3 neutropenia and grade 3 anemia appeared, but resolved within a few days (12). The patient underwent colonoscopy following recovery from DIC. A sigmoid colon tumor was detected (Fig. 2B) and biopsy of the tumor revealed poorly differentiated adenocarcinoma (Fig. 1B), without mutations of the RAS, BRAF or PIK3CA oncogenes. On immunohistochemical analysis, the tumor cells were negative for CK7 and positive for CK20 and CDX2. The patient was treated with 3 cycles of mFOLFOX6, followed by 5 cycles of CapeOX (oral capecitabine at 2,000 mg/day on days $1-14$ and intravenous oxaliplatin at $130 \mathrm{mg} / \mathrm{m}^{2}$ on day 1 , every 3 weeks), achieving shrinkage of the tumor. However, a new lesion was detected at the ninth thoracic vertebra on CT after 150 days of treatment. Subsequently, combination chemotherapy using irinotecan and panitumumab was initiated. The PS improved, but progression of the primary lesion and lymphadenopathy was observed after 8 cycles. Although third-line treatment with trifluridine/tipiracil hydrochloride (110 mg/day per os on days 1-5 and 8-13, every 4 weeks) was initiated, the disease progressed and the patient succumbed to the disease 10 months after the diagnosis. Informed consent was obtained from the patient for the publication of the case details prior to treatment initiation.

\section{Discussion}

Malignant solid tumors may invade the bone marrow, particularly in patients with cancer of the breast, stomach and prostate gland (1). Only a small percentage of breast cancer cases present with bone marrow metastasis at initial diagnosis, but $36 \%$ develop this complication during the course of the disease (13). Poorly differentiated adenocarcinoma or signet ring cell carcinoma of the stomach often invade the bone marrow, and $1.4 \%$ of surgically resected gastric cancers were reported to exhibit bone marrow invasion (14). Similar to the present case, patients with bone marrow metastases from breast or gastric cancer tend to develop DIC. The main treatments for this disease are considered to be chemotherapy and intensive supportive therapy. Reliable molecular markers predictive of bone marrow metastasis have yet to be identified.

Bone marrow metastasis from CRC is rarely observed, with only 10 cases of CRC with bone marrow metastasis reported to date (6). Although $24 \%$ of autopsy cases of CRC were reported to have bone marrow metastases, they may not be apparent on standard radiological examination and as a coagulation disorder. In cases with clinically apparent bone marrow metastases of CRC in association with DIC, chemotherapy was mainly performed against the tumor, with intensive supportive therapy for DIC appended. The majority of the reported cases employed combination chemotherapy with FOLFOX plus molecular-targeted agents, but survival was generally poor ( $\leq 7$ months) (6). One of the reasons for 

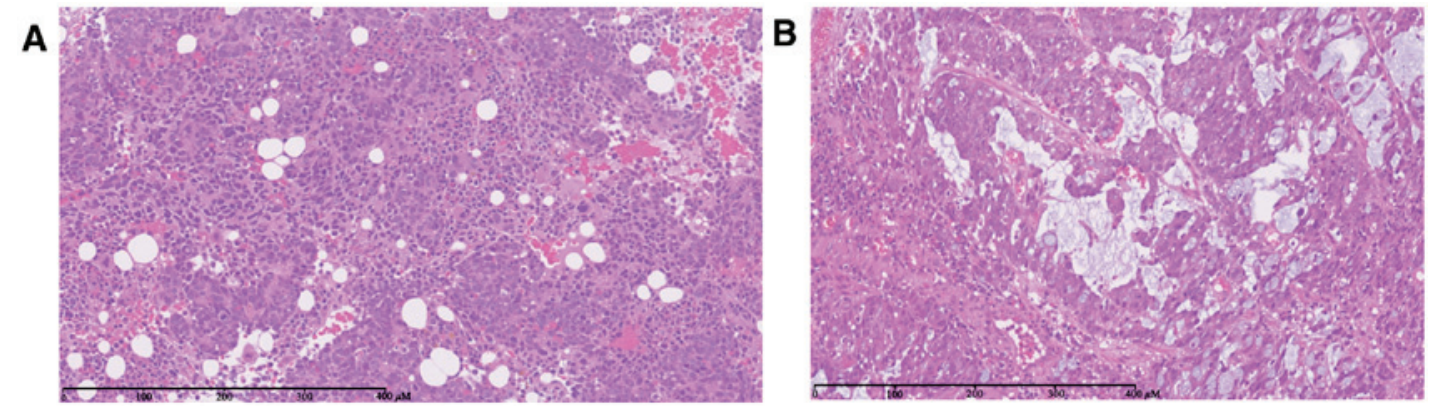

Figure 1. Histological examination of (A) aspiration biopsy from the bone marrow and (B) the biopsy specimen from the primary sigmoid colon adenocarcinoma. Hematoxylin and eosin staining; bar, $400 \mu \mathrm{m}$.
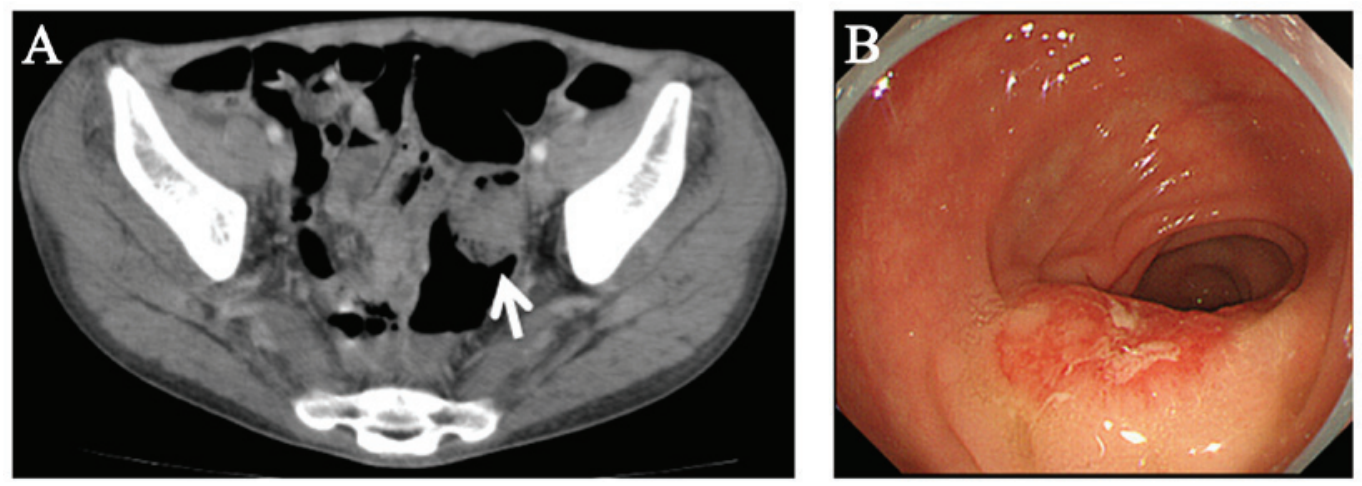

Figure 2. (A) Computed tomography at initial diagnosis revealed a tumor in the sigmoid colon (arrow). (B) Colonoscopy 14 days after the initiation of chemotherapy revealed an ulcerated, non-obstructive tumor in the sigmoid colon.

unfavorable survival was suggested to be the poor general condition of the patients, as DIC hinders the administration of sufficiently intensive chemotherapy. Another possibility may be primary resistance of the CRC cells to chemotherapy as a common characteristic of bone marrow metastasis; however, the molecular mechanisms underlying the resistance of the CRC cells have not been elucidated.

A specific explanation as to why bone metastases often occur in cancers of the breast and stomach, but not in cancer of the large bowel, has yet to be provided. On histological examination of the present case, the primary tumor was a poorly differentiated adenocarcinoma with mucin production, whereas the metastasized cells in the bone marrow exhibited the characteristics of moderately to poorly differentiated adenocarcinoma, suggesting that tumor cells from different lesions displayed similar histological characteristics. There have been a few reports on histological and molecular biological analyses of CRC cells metastasizing to the bone marrow. A unique case of CRC metastasis to the bone marrow reported by Shah et al involved a poorly differentiated adenocarcinoma of the ascending colon, with a CK7-positive, CK20-negative phenotype on immunohistochemical analysis, wild-type $K R A S$ and positive for a $B R A F$ V600E mutation (6). CRCs with the $B R A F$ V600E mutation are known to respond poorly to chemotherapy and are closely associated with certain characteristics, including location in the right colon, hypermutation, a microsatellite-unstable type and mucinous pattern (15). These characteristics support the hypothesis that a trait of bone marrow metastasis may be involved.
Although our case also exhibited mucinous histology at the primary site, wild-type $R A S, B R A F$ and $P I K 3 C A$ genes were detected in the CRC cells. Wild-type $R A S$ and $B R A F$ are favorable predictive factors of anti-epidermal growth factor receptor (EGFR) treatment for CRC (16), whereas CRC harboring mutations of $P I K 3 C A$ and wild-type $R A S$ is resistant to anti-EGFR drugs (17). However, the association between bone marrow metastasis and tumor type, such as gene mutations, was unclear.

Cytokeratin is an intermediate filament protein, mainly detected in differentiated epithelial cells. In the cytoplasm, intermediate filament proteins play a role in supporting cell morphology through attachment to desmosomes and hemidesmosomes. Moreover, it was recently demonstrated that intermediate filament proteins were able to bind to signaling proteins and modulate their activity (18). The majority of breast cancers easily metastasize to the bone marrow and express CK7, but not CK20. Conversely, CRC cells express CK20, but not CK7, and a CK7-positive, CK20-negative phenotype reportedly appears in only $2 \%$ of CRCs (19-20). As mentioned above, Shah et al reported that CK7-expressing CRCs exhibited a bone marrow-invading phenotype (6); however the present case exhibited a common CK7-negative, CK20-positive phenotype. Interestingly, in this case there was also an increased level of CYFRA. CYFRA is a fragment of CK19 and is often increased in lung squamous cell carcinoma, ovarian cancer and breast cancer, but not in colorectal cancer. Pierga et al reported that elevated levels of CYFRA correlated with detection of micrometastasis of breast cancer to the bone 
marrow (21). Although the underlying mechanisms remain unclear, different $\mathrm{CK}$ expressions may affect signaling pathways in CRC cells leading to bone marrow metastasis.

CDX2 is a major regulator of intestine-specific genes involved in cell growth and differentiation and plays a role in early embryonic development of the intestinal tract. A recent study reported longer relapse-free survival of stage II/III CDX2-positive CRC (22). The present case displayed CDX2-positive CRC cells, but whether CDX2 is associated with survival in stage IV CRC and with bone marrow metastasis has not been elucidated.

Compared with previous reported cases, the treatment in our patient exhibited favorable efficacy, possibly because the patient was relatively young and in a good general condition, experienced no serious complications associated with organ metastases, and was able to tolerate a full course of FOLFOX therapy and intensive supportive therapy for DIC. Although several CRC patients with bone marrow metastasis were treated with the anti-EGFR antibody cetuximab (9), the effects of cetuximab were not clearly demonstrated. In the subsequent therapy of this patient, panitumumab was beneficial and the therapy was continued for 8 cycles.

We herein reported a rare case of sigmoid colon cancer harboring bone marrow metastasis in association with DIC. The immunohistochemical and molecular biological characteristics of the CRC differed from those in previously reported cases. Further research is required to elucidate the mechanisms of bone marrow metastasis of CRC and to establish efficient biomarkers that are able to predict a high risk of bone marrow invasion and coagulation disorder.

\section{Acknowledgements}

We would like to thank Dr Shinya Oda (Department of Cancer Biology, National Hospital Organization Kyushu Cancer Center) and the medical staff of the National Hospital Organization Kyushu Cancer Center for their contribution to patient diagnosis and treatment.

\section{References}

1. Papac RJ: Bone marrow metastases. A review. Cancer 74: 2403-2413, 1994.

2. Hayes DF: Prognostic and predictive factors revisited. Breast 14: 493-499, 2005

3. Kobayashi M, Okabayashi T, Sano T and Araki K: Metastatic bone cancer as a recurrence of early gastric cancer-characteristics and possible mechanisms. World J Gastroenterol 11: 5587-5591, 2005.

4. Wiedswang G, Borgen E, Kåresen R, Kvalheim G, Nesland JM, Qvist H, Schlichting E, Sauer T, Janbu J, Harbitz T and Naume B: Detection of isolated tumor cells in bone marrow is an independent prognostic factor in breast cancer. J Clin Oncol 21: 3469-3478, 2003

5. Haggar FA and Boushey RP: Colorectal cancer epidemiology: Incidence, mortality, survival, and risk factors. Clin Colon Rectal Surg 22: 191-197, 2009.

6. Shah SM, Rosenthal MH, Griffin GK, Jacobsen ED and McCleary NJ: An aggressive presentation of colorectal cancer with an atypical lymphoproliferative pattern of metastatic disease: A case report and review of the literature. Clin Colorectal Cancer 13: e5-e11, 2014.
7. Lee JL, Lee JH, Kim MK, Cho HS, Bae YK, Cho KH, Bae SH, Ryoo HM, Lee KH and Hyun MS: A case of bone marrow necrosis with thrombotic thrombocttopenic purpura as a manifestation of occult colon cancer. Jpn J Clin Oncol 34: 476-480, 2004.

8. Pleyer L, Went P, Russ G, Prinz E, Faber V, Röwert HJ, Karlbauer R and Greil R: Massive infiltration of bone marrow in colon carcinoma after treatment with activated protein $\mathrm{C}$. Wiener Klinische Wochenschrift 119: 254-258, 2007.

9. Wang YC, Chang PY and Yao NS: Bone marrow necrosis caused by metastatic colon cancer. J Clin Oncol 27: e48, 2009.

10. Song JY and Dwyre DM: Metastatic adenocarcinoma involving the bone marrow. Blood 120: 3170, 2012.

11. Wada H, Gabazza EC, Asakura H, Koike K, Okamoto K, Maruyama I, Shiku H and Nobori T: Comparison of diagnostic criteria For Disseminated Intravascular Coaglation (DIC): Diagnostic criteria of the International Society of Thrombosis and Hemostasis (ISTH) and of the Japanese Ministry of Health and Welfare for Overt DIC. Am J Hematol 74: 17-22, 2003.

12. National Cancer Institute: Common Terminology Criteria for Adverse Events v4.0. NIH Publication: Number 10-5410, 2010.

13. Braun S, Pantel K, Müller P, Janni W, Hepp F, Kentenich CR, Gastroph S, Wischnik A, Dimpfl T, Kindermann G, et al: Cytokeratin-positive cells in the bone marrow and survival of patients with stage I, II, or III breast cancer. N Engl J Med 342: 525-533, 2000.

14. Iguchi H: Recent aspects for disseminated carcinomatosis of the bone marrow associated with gastric cancer: What has been done for the past, and what will be needed in future? World J Gastroenterol 21: 12249-12260, 2015.

15. Pai RK, Jayachandran P, Koong AC, Chang DT, Kwok S, Ma L, Arber DA, Balise RR, Tubbs RR, Shadrach B and Pai RK: BRAF-mutated, microsatellite-stable adenocarcinoma of the proximal colon: An aggressive adenocarcinoma with poor survival, mucinous differentiation, and adverse morphologic features. Am J Surg Pathol 36: 744-752, 2012.

16. Therkildsen C, Bergmann TK, Henrichsen-Schnack T, Ladelund $S$ and Nilbert $M$ : The predictive value of KRAS, NRAS, BRAF, PIK3CA and PTEN for anti-EGFR treatment in metastatic colorectal cancer: A systematic review and meta-analysis. Acta Oncol 53: 852-864, 2014.

17. Sartore-Bianchi A, Martini M, Molinari F, Veronese S, Nichelatti M, Artale S, Di Nicolantonio F, Saletti P, De Dosso S, Mazzucchelli L, et al: PIK3CA mutations in colorectal cancer are associated with clinical resistance to EGFR-targeted monoclonal antibodies. Cancer Res 69: 1851-1857, 2009.

18. Coulombe PA and Wong P: Cytoplasmic intermediate filaments revealed as dynamic and multipurpose scaffolds. Nat Cell Biol 6: 699-706, 2004

19. Bayrak R, Yenidünya S and Haltas H: Cytokeratin 7 and cytokeratin 20 expression in colorectal adenocarcinomas. Pathol Res Pract 207: 156-160, 2011.

20. Park SY, Kim BH, Kim JH, Lee S and Kang GH: Panels of immunohistochemical markers help determine primary sites of metastatic adenocarcinoma. Arch Pathol Lab Med 131: 1561-1567, 2007.

21. Pierga JY, Deneux L, Bonneton C, Vincent-Salomon A, Nos C, Anract P, Magdelénat H, Pouillart P and Thiery JP: Prognostic value of cytokeratin 19 fragment (CYFRA 21-1) and cytokeratin-positive cells in bone marrow samples of breast cancer patients. Int J Biol Markers 19: 23-31, 2004.

22. Dalerba P, Sahoo D, Paik S, Guo X, Yothers G, Song N, Wilcox-Fogel N, Forgó E, Rajendran PS, Miranda SP, et al: CDX2 as a prognostic biomarker in stage II and stage III colon cancer. N Engl J Med 374: 211-222, 2016. 